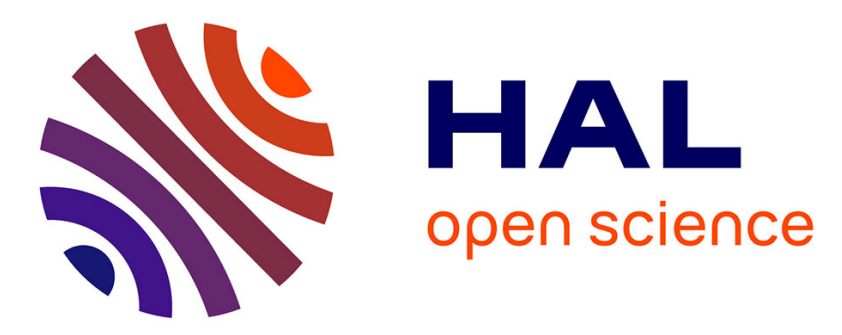

\title{
DYNAMIQUES CONJUGALES ET DYNAMIQUES INTERGÉNÉRATIONNELLES DANS L'IMMIGRATION MAROCAINE EN FRANCE
}

Catherine Delcroix

\section{- To cite this version:}

Catherine Delcroix. DYNAMIQUES CONJUGALES ET DYNAMIQUES INTERGÉNÉRATIONNELLES DANS L'IMMIGRATION MAROCAINE EN FRANCE. Migrations Société, 2013, Les migrations au regard des récits de vie, 25 (145), pp.79-90. hal-01287683

\section{HAL Id: hal-01287683 \\ https://hal.science/hal-01287683}

Submitted on 14 Mar 2016

HAL is a multi-disciplinary open access archive for the deposit and dissemination of scientific research documents, whether they are published or not. The documents may come from teaching and research institutions in France or abroad, or from public or private research centers.
L'archive ouverte pluridisciplinaire HAL, est destinée au dépôt et à la diffusion de documents scientifiques de niveau recherche, publiés ou non, émanant des établissements d'enseignement et de recherche français ou étrangers, des laboratoires publics ou privés. 


\section{PEGARDOS DYNAMIQUES CONJUGALES ET

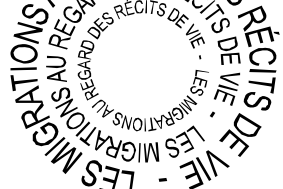 DYNAMIQUES INTERGÉNÉRA- TIONNELLES DANS L'IMMIGRATION MAROCAINE EN FRANCE}

Catherine DELCROIX *

Dans la présente contribution nous allons nous pencher sur les formes familiales et les dynamiques conjugales de couples marocains ayant émigré vers la France dans les années 1960 et 1970, en nous intéressant à cette génération de parents restée longtemps silencieuse et invisible. Alors que leur décision de quitter le Maroc pour vivre en France s'inscrivait dans un projet de mobilité et de réussite sociale, ces couples ont été et sont toujours confrontés à d'importants défis sociaux et éducatifs, et il leur a fallu faire face aux crises économiques et familiales liées à un accident du travail, à la perte d'emploi et au manque de ressources financières. Ces crises sont aussi liées aux difficultés rencontrées par leurs enfants pour se construire un avenir en étant issus du monde ouvrier, en étant confrontés au racisme et à la disparition des emplois industriels. Elles sont liées, enfin, à la gestion des "papiers", devenue de plus en plus contraignante au fil des décennies. Changer de pays impose à ces couples de nombreuses remises en question, notamment en matière de division traditionnelle des rôles entre hommes et femmes, et les relations intergénérationnelles sont soumises à rude épreuve : les migrants ont dû faire des apprentissages et procéder à des adaptations, ils ont vécu des recompositions et des ruptures.

Notre objectif est ici de donner à ces parents une visibilité, voire une légitimité, en montrant comment ils font face à des situations diverses et complexes, en soulignant leur ténacité et leur créativité dans la résolution de crises conjugales et familiales. Ils sont toujours à la recherche de stratégies capables de les aider à surmonter les obstacles liés à la précarité et au déracinement. Comment ont-ils réussi à concilier une vie professionnelle de travail pénible et une vie de famille? La recherche de la réussite matérielle se fait-elle aux dépens de l'équilibre familial et conjugal ? Par ailleurs, l'histoire de ces couples est aussi celle d'une

* Professeur de sociologie, Laboratoire Cultures et Sociétés en Europe, Université de Strasbourg, Maison interdisciplinaire des sciences de l'homme d'Alsace (MISHA). 
génération charnière entre un "ici" et un "là-bas". Ils sont aujourd'hui parents et grands-parents d'enfants eux-mêmes devenus adultes et citoyens français dans leur majorité. Ils ont bien souvent des frères et des sœurs qui ont émigré vers d'autres pays européens: ils peuvent alors comparer les divers parcours, les éventuels échecs et adapter leur conduite en conséquence. Lorsque les familles sont éclatées entre plusieurs pays européens, cela induit une diversification des pratiques, mais aussi des formes de rivalité par rapport au projet de mobilité. Comment garder alors l'esprit de famille au-delà de ces différences? Comment concilier aussi réalités du pays dans lequel on vit et pressions de la famille et de la société du pays d'origine? Ces adultes, arrivés à l'âge des bilans, sont détenteurs de clés de compréhension et de ressources pour donner du sens à leurs actions, ressources indispensables à leurs enfants et petits-enfants appelés à innover pour faire face à ce que Claudine Attias-Donfut et François-Charles Wolff appellent le " désenchaînement des générations ". En effet, "les immigrés sont des pionniers. Cette position unique et inimitable leur interdit de s'ériger en modèle. Ce sont leurs enfants qui vont fonder une nouvelle lignée, euxmêmes n'en sont que les passeurs $1{ }^{1}$.

Comprendre le rôle de passeurs de ces parents primo-migrants, cette transmission intergénérationnelle et cette médiation entre un "ici" et un "là-bas", suppose de solliciter le récit rétrospectif de leur vie - des deux côtés des rives de la Méditerranée, avant et après l'arrivée en France - à un moment clé de leur existence, celui de l'autoévaluation de leur parcours. Outre leur dimension historique, les narrations de soi donnent à voir l'intrication des domaines de la vie, l'enchevêtrement des logiques individuelles et sociales inscrites dans des contextes dans des périodes et des espaces précis. Elles permettent notamment de repérer les événements biographiques, tels les accidents du travail qui révèlent les conditions de travail faites aux migrants, qui font non seulement basculer ces pères et leurs familles dans la précarité, mais qui peuvent avoir des conséquences sur les configurations conjugales et familiales, sur les rapports de genre et intergénérationnels. Elles donnent accès aux solutions que ces hommes et ces femmes ont inventées et expérimentées pour s'en sortir. Le faire ressortir, tel est aussi le propos de notre contribution.

Pour mieux connaître les dynamiques conjugales et familiales de ces couples, nous nous appuyons sur les résultats de différentes enquêtes

1. ATTIAS-DONFUT, Claudine ; WOLFF, François-Charles, Le destin des enfants d'immigrés : un désenchaînement des générations, Paris : Éd. Stock, 2009, 320 p. (voir p. 28). 
menées auprès de familles vivant en situation précaire dans des "cités" de villes françaises". Ces "cités", composées de logements sociaux, forment des quartiers qui sont engagés dans des programmes de développement social et urbain fondés sur des politiques spécifiques, regroupées sous l'expression "politique de la ville"3. Notre contribution ne concerne donc pas toutes les familles marocaines installées en France, mais uniquement celles qui vivent dans ce type de contexte. Ces parents ont été amenés à éduquer leurs enfants avec l'aide de professionnels, mais ont été aussi très contrôlés ${ }^{4}$. En effet, par le biais de ces rencontres avec des enseignants, des travailleurs sociaux, ils se sont constitués des réseaux qui leur ont apporté un soutien tout en étant contraignants.

Ces recherches ont eu recours à la méthodologie de la "biographical policy evaluation " ${ }^{5}$, que l'on peut traduire par "évaluation biographique des politiques publiques". II s'agit de témoignages biographiques recueillis pour mettre en évidence les forces et les faiblesses des politiques publiques en ce qui concerne leurs effets sur la vie quotidienne, un des aspects traités étant les équilibres familiaux. Parents et enfants ont été interviewés séparément et ensemble. Il s'agissait de reconstituer des "historiques de famille" en suivant les étapes de leur vie.

2. Cf. DELCROIX, Catherine, "Des récits croisés aux histoires de familles", Current Sociology/ Sociologie Contemporaine, vol. 43, décembre 1995, pp. 61-67 ; DELCROIX, Catherine, "Les parents des cités : la prévention familiale des risques encourus par les enfants", Annales de la Recherche Urbaine, $\mathrm{n}^{\circ}$ 83-84, 1999, pp. 97-107 ; DELCROIX, Catherine, "Discrédit et action collective. La lutte d'une association de 'pères musulmans'", in : COSSÉE, Claire ; LADA, Emmanuelle ; RIGONI, Isabelle (sous la direction de), Faire figure d'étranger : regards croisés sur la production de l'altérité, Paris : Éd. Armand Colin, 2004, pp. 191-210; DELCROIX, Catherine (sous la direction de), Étude exploratoire de méthodologies de participation des habitants à l'opération de renouvellement urbain de l'agglomération drouaise (28), Laboratoire Printemps, Université Versailles Saint-Quentin, pour l'Agence nationale de rénovation urbaine, mars 2006 mai 2008.

3. Depuis le début des années 1980, les gouvernements successifs ont élaboré et mis en œuvre une "politique de la ville", c'est-à-dire une politique publique globalisante, partenariale, transversale et territorialisée. L'objectif en est de remédier aux difficultés socioéconomiques des habitants en incitant à décloisonner l'activité des différentes institutions (municipalité, écoles, agences pour l'emploi, associations, police) en les amenant à travailler ensemble au niveau local. Cette synergie peut faciliter l'aide apportée aux personnes, mais les informations à leur sujet étant ainsi rassemblées, elle permet aussi un plus grand contrôle des conduites. Les pratiques éducatives sont devenues un enjeu central car aux yeux des autorités, c'est par elles que passe la prévention de la délinquance.

4. Voir aussi LÉOMANT, Christian ; SOTTEAU-LÉOMANT, Nicole, Aide contrainte et citoyenneté, justice des mineurs et intervention éducative en milieu ouvert. Rapport de recherche, Paris : GERS-CNRS, Ministère de la Justice, 2001, 122 p.

5. APITZSCH, Ursula ; INOWLOCKI, Lena ; KONTOS Maria, "The method of biographical policy evaluation", in : APITZSCH, Ursula ; KONTOS, Maria (Eds.), Self-employment activities of women and minorities : their success or failure in relation to social citizenship policies, Wiesbaden, VS Verlag für Sozialwissen-schaften/Springer Science and Business Media, 2008, pp. 12-18. 
En outre, nous nous basons aussi sur une étude de cas inscrite dans la durée, celle d'une famille marocaine installée en France depuis plus de 30 ans $^{6}$. Les parents ont eu huit enfants, six garçons et deux filles. Après avoir interviewé environ 200 familles, dont une cinquantaine d'origine marocaine, il nous a paru intéressant d'approfondir l'étude de cette famille qui partageait avec beaucoup d'autres des situations liées à la précarité et au parcours migratoire. Nous avons rencontré ses membres à de nombreuses reprises pendant les six années durant lesquelles nous les avons suivis, en vivant par moments avec eux. II s'agissait d'ajouter aux entretiens croisés les résultats d'une observation de longue durée, en particulier dans la vie quotidienne. Pour aller au-delà de l'identification de logiques d'action idéal-typiques, nous voulions restituer la dynamique des rapports de genre et des rapports intergénérationnels et intragénérationnels au sein de cette famille.

Pour l'ensemble des familles étudiées à l'occasion des différentes recherches, nous décrirons d'abord les formes familiales à l'arrivée en France, puis nous envisagerons les processus de dissociation entre couple parental et couple conjugal, et enfin les effets des crises familiales sur la parentalité et la conjugalité.

\section{Formes familiales à l'arrivée en France}

Les hommes sont d'abord venus seuls s'embaucher comme ouvriers dans le bâtiment, l'agriculture, le nettoyage industriel ou l'automobile... Ils ont travaillé dans des secteurs caractérisés par la pénibilité, les hauts risques d'accidents et le peu de qualification des emplois tenus. Ils changeaient souvent d'entreprises, faisaient des séjours dans le pays d'origine pour y retrouver leurs familles et récupérer de leur fatigue. Dès la fin des années 1960 et le début des années 1970, en France comme ailleurs en Europe, des mesures visant à restreindre l'immigration sont mises en place ${ }^{7}$. Par la suite, les évolutions économiques mondiales résultant de la décision américaine du 15 août 1971 de mettre fin à la convertibilité du dollar en or conduiront progressivement aux licenciements et aux délocalisations. C'est dans ce contexte que le 6 juillet 1974 le gouvernement français décide de suspendre l'immigration des tra-

6. Cf. DELCROIX, Catherine, Ombres et lumières de la famille Nour : comment certains résistent face à la précarité, Paris : Éd. Payot, 2001, 258 p.

7. Circulaire du 29 juillet 1968 supprimant, sauf quelques exceptions, la procédure de régularisation pour les travailleurs non qualifiés ; circulaires dites "Marcellin-Fontanet" des 23 février 1972 et 15 septembre 1972 liant le recrutement de travailleurs étrangers à la situation du marché du travail et subordonnant la possession d'une carte de séjour à la détention d'un emploi. 
vailleurs étrangers non ressortissants de la Communauté économique européenne.

Ce changement de la réglementation française sur l'immigration conduit les immigrés présents en France à revoir de fond en comble leurs stratégies de vie. La France n'accepte plus de nouveaux immigrés, mais ceux qui sont déjà installés pourront de nouveau, dès 1976, faire venir leur femme et leurs enfants 8 . Dans les années 1970 et 1980, les femmes marocaines, qui avaient émigré pour suivre leurs maris, sont très majoritairement soit berbères venant de villages du Moyen-Atlas, soit originaires d'Agadir et de ses environs, soit originaires des régions frontalières de l'Algérie. À leur arrivée en France, elles sont analphabètes ou faiblement instruites, ne parlent pas forcément le français et sont souvent peu habituées au mode de vie citadin.

Petit à petit, cette migration de peuplement met fin aux migrations tournantes, des situations où les travailleurs restent séparés de leur famille, et qui perdurent aujourd'hui dans le secteur agricole. C'est ce que rapporte Ouarda, une jeune femme marocaine de 30 ans, qui s'est mariée en France avec un Français d'origine marocaine : "I Mon père restait huit mois en France avec un contrat agricole et quatre mois au Maroc. On n'est pas resté beaucoup avec papa. On connaissait la France de loin. Et puis il a eu un accident du travail. On a pris un avocat, tout a été conçu pour l'exploiter. C'est le patron qui décide s'il peut ou non régulariser le travailleur en France. Mes parents et nous [elle, ses deux frères et sa sœur], nous n'étions jamais en sécurité ! J'ai quand même décidé de me marier en France et i'ai aidé mon père pour ses papiers. II avait amené avec lui un peu de la France chez lui, au Maroc II.

8. En 1996, les Marocains vivant en France sont au nombre de 516000 (SOPEMI, Tendances des migrations internationales. Rapport annuel 1996, Paris : OCDE, 1997, pp. 67-70). De plus, comme le souligne Salah Ferhi, le nombre des personnes d'origine maghrébine ayant ou non acquis la nationalité française et vivant en France « atteint facilement les 4 millions ». FERHI, Salah, "L'immigration arabe dans le monde", Migrations Société, vol. 21, n 125, septembreoctobre 2009, pp. 11-40 (voir p. 16). 


\section{Identités recomposées ou repli sur la famille et nos- talgie du pays d'origine}

Les couples s'installent pour de bon et créent leur ménage dans un univers inconnu. Leurs descendants naissent en nombre important ${ }^{9}$. Les questions de la conjugalité mais aussi de la perpétuation de la lignée et des transmissions se posent différemment dans le contexte migratoire. Ces hommes et ces femmes fondent une famille nucléaire. Prenons l'exemple d'Amin, le père de la famille Nour que nous avons longuement suivie. Au moment des premiers entretiens, Amin et sa femme Diamila sont respectivement âgés de 58 ans et de 45 ans. Ils ont eu ensemble huit enfants. Amin raconte qu'il travaille en France depuis 1969 lorsqu'il décide en 1974 de faire venir du Maroc sa jeune épouse : II J'ai fait le passeport touriste. Je l'ai amenée ici. Quand i'ai fait le contrat, le bureau de la main-d'œuvre m'a dit qu'il y avait pas de logement et qu'il fallait que ma femme retourne au Maroc. Je suis allé à l'Office des migrations, j'ai fait une demande à la préfecture pour que ma femme ait le droit de rester [Djamila était enceinte]. La préfecture a accepté 11 . Alors demandeur d'emploi, il accepte rapidement le seul travail qu'il trouve. "I À la fin 1974, il n'y avait pas de travail, alors i'ai dit d'accord à un patron qui habitait loin. Je prenais le train à quatre heures du matin et je restais à la gare jusqu'à huit heures avant de commencer sur le chantier, et le soir je rentrais chez moi vers 21 heures II. Une fois installée dans un appartement situé au cœur d'une cité, Diamila a dû s'adapter : "I Quand je suis venue ici, je pleurais, i'avais perdu ma famille. Je ne savais pas parler français... "I.

Comme d'autres épouses se retrouvant dans la même situation, elle se concentre sur sa fonction de mère, se sent reconnue, appréciée, ce qui permet un certain dialogue entre elle et son mari : " Ça s'est bien passé. II ne voulait pas me laisser sortir dehors, mais quand même... le samedi et le dimanche, on sortait. On partait chez la famille de loin, on allait faire des visites, des musées, on partait au Jardin des plantes II. Elle a le désir de s'ouvrir à une nouvelle société et le fait que le couple ait dû ajuster ses comportements afin d'être en règle avec l'administration ${ }^{10}$

9. “Entre 1969 et 1990, d'après l'Office des migrations internationales (OMI), il est entré dans le cadre du regroupement familial 140635 personnes. L'un des résultats d'un tel phénomène est le rajeunissement de cette immigration. Ainsi, en 1990, les moins de vingt ans représentaient $32 \%$ de la population totale et les moins de quarante ans, 55,8\% ». ZEHRAOUI, Ahsène, "Les Algériens, de la migration à l'installation", in : DEWITTE, Philippe (sous la direction de), Immigration et intégration : l'état des savoirs, Paris : Éd. La Découverte, 1999, pp. 121-127 (voir p. 122).

10. Le père devant rester chez le même patron pour ne pas risquer de perdre l'autorisation de séjour en France pour lui et sa famille. 
a été l'occasion de nombreux échanges. Amin devait travailler y compris le samedi et Diamila s'occupait seule de leurs enfants. Cela a commencé à souder leur relation. II comptait sur elle, elle comptait sur lui. Un respect mutuel s'est installé. Le projet commun de faire leur vie en France de façon définitive fut pour eux, comme pour tous les couples poursuivant un but semblable, source d'équilibre et de dynamisme. Avec le temps et la montée du chômage ouvrier, certaines femmes sont entrées sur le marché du travail, ce qui $a$, comme nous le verrons ciaprès, beaucoup transformé les rapports conjugaux et familiaux.

Au sein d'autres couples, des épouses très isolées définissent, selon les propos d'Eva Lorenzoni, II leur appartenance essentiellement par rapport au passé, en construisant une identité liée aux origines et en continuant à rêver de retourner au Maroc, même si la présence des enfants - qui considèrent [...] la France comme leur pays - leur font prendre conscience que ce rêve ne deviendra jamais une réalité. "Mon pays reste le Maroc", "mon cœur est marocain", nous ont dit les femmes constituant ce groupe et qui emploient en permanence dans leurs discours l'expression "chez nous" pour indiquer la manière dont se passent les choses au sein de la communauté des compatriotes, en opposition aux modèles et aux styles de vie des Italiens et des Français, qui à leurs yeux restent fondamentalement étrangers $1{ }^{11}$.

Les projets des conjoints ne sont pas toujours en harmonie et évolvent dans le temps. Même s'il y avait au départ la volonté de fonder avant tout un couple parental dont l'objectif en migrant est d'élargir le "champ des avenirs possibles" pour leurs enfants, ils n'ont pas nécessairement la même position sur les rapports et le type de distance à avoir avec le "ici" et le "là-bas".

La question des transmissions culturelles, notamment, fait beaucoup débat. En effet, contrairement aux familles françaises, les familles marocaines ont été mises à rude épreuve. Les familles françaises savent pouvoir compter sur l'action quotidienne de l'école, voire des médias, pour contribuer à la socialisation de leurs enfants qui finiront par leur ressembler sans qu'elles aient à faire aucun effort conscient. II n'en est pas du tout de même pour les parents émigrés/immigrés. Venus d'ailleurs, ayant une autre culture, souvent une autre religion, ils ont longtemps

11. LORENZONI, Eva, "Appartenance identitaire en migration : Marocaines en France et en Italie", Migrations Société, vol. 21, n 123-124, mai-août 2009, pp. 225-239 (voir p. 230). 
été seuls à essayer d'en transmettre quelque chose à leurs enfants, dans des conditions très difficiles ${ }^{12}$.

Quelle langue doit-on parler en famille? Comment pratiquer et transmettre l'appartenance religieuse? Faut-il l'imposer ou pas? Comment traiter en France du contrôle de la sexualité des filles, si importante pour l'honneur familial, et des rapports hommes-femmes qui, dans la société traditionnelle marocaine, sont du ressort de la responsabilité familiale? Autant de questions qui amènent ces femmes et ces hommes à s'interroger sur les rapports entre couple parental et couple conjugal.

\section{Couple parental et couple conjugal : effets des crises sur la parentalité et la conjugalité}

Parmi les couples rencontrés, beaucoup ont commencé leur vie maritale sur la base d'une division traditionnelle et patriarcale des rôles. Leurs unions avaient été majoritairement arrangées au sein de leurs familles respectives, et certaines ont été conclues sans le consentement de l'épouse. L'installation en France a profondément modifié les rapports conjugaux. Vivre en terre étrangère, souvent sans l'appui de la famille, requiert un travail d'équipe qui implique la distribution des rôles en fonction des difficultés à affronter.

Au départ, il n'y avait pas de séparation entre couple parental et conjugal. Le mariage s'est fait autour du partage d'un intérêt collectif, selon le modèle du patriarche qui ramène l'argent, qui a les contacts avec l'extérieur, qui exerce l'autorité... Ce modèle est remis en cause suite à des accidents du travail, de la mise au chômage pour des pères... et à la nouvelle situation des femmes qui investissent le monde du travail. Fatiha, âgée de 50 ans au moment de l'entretien et mère de dix enfants, explique ainsi la transformation de sa vie de femme et d'épouse : "I Ne pas avoir choisi son mari et ne pas avoir été à l'école, c'est deux choses... Je mourrai avec [...]. Mes filles ont choisi leur mari. Je voulais qu'elles fassent des études. Je travaille pour les aider. J'ai encore huit enfants à la maison [...]. Moi, i'ai toujours voulu bouger, i'ai fait des formations, des stages. Je ne voulais pas rester à la maison, je voulais sortir, voir du monde. Mon mari ne voulait pas mais je l'ai fait. Je travaille au lycée pour nettoyer les classes. J'ai fait des remises à niveau, de l'alphabétisation 11.

12. Cf. BERTAUX, Daniel; DELCROIX, Catherine, "Transmissions familiales et mobilités", introduction au dossier "Transmissions familiales en migration", Migrations Société, vol. 21, $\mathrm{n}^{\circ}$ 123-124, mai-août 2009, pp. 89-96. 


\section{Les accidents du travail, des moments charnières dans la vie des couples rencontrés}

Dans les années 1980, l'instabilité du marché du travail force beaucoup des hommes rencontrés, tel Amin, à changer de nombreuses fois d'employeur. Malgré cela il avait un bon salaire, ce qui rendait le climat familial favorable.

En juin 1980, alors qu'Amin a 40 ans, il est victime d'un accident du travail. En 1982 il en a un deuxième : II Je rangeais la place de l'ascenseur. J'étais sur l'échafaudage et je suis tombé du deuxième étage dans la cage d'ascenseur. J'ai eu une fracture du talon et je suis resté quatre mois hospitalisé 11 . Ce deuxième accident, survenu comme le précédent sur un chantier mais plus invalidant, est inscrit dans un parcours professionnel que nous avons constaté chez d'autres pères de famille enquêtés. II ne semble pas lié au manque de conditions de sécurité de l'entreprise où Amin travaillait, mais plutôt au fait que depuis l'adolescence il travaillait sans compter ses forces et dans des conditions souvent extrêmes.

À son arrivée en France, en 1969, Amin doit trouver un patron qui accepte de faire les démarches administratives nécessaires pour le sortir de la situation irrégulière au regard du séjour. En contrepartie, celui-ci exige qu'il reste son ouvrier pour un salaire très faible pendant plus de trois ans après sa régularisation: II Je ne sais pas, se souvient Amin, on ne comptait pas les heures. Jusqu'à minuit... non, de sept heures du matin à sept heures du soir. On arrêtait une heure à midi. J'ai passé trois ans avec ce patron-là. On a fait une villa. On est rentré dans la villa. On n'avait pas de lit, juste des planches $1 /$.

Dans les années 1960, on trouve beaucoup d'immigrés, tel Amin, dans des petites entreprises du bâtiment ne respectant pas la législation du travail. Ils sont en situation irrégulière, ce qui les rend particulièrement dépendants : "L'articulation de l'exploitation directe et de la précarité juridique autorise à parler d'un "système de travail migrant". Les conditions de travail extrêmes, associées à des modes d'habitat insalubres, entraînent effectivement une dégradation rapide de la santé $1{ }^{13}$. À travers le récit d'Amin et de ses proches, nous découvrons comment, en une dizaine d'années, précédées par son activité de berger et d'agriculteur au Maroc, il voit son capital-santé s'affaiblir et sa vigilance diminuer, ce qui va le conduire inexorablement à être victime d'un accident du travail.

13. TRIPIER, Maryse, L'immigration dans la classe ouvrière en France, Paris : Éd. CIEMI - L'Harmattan, 1990, $336 \mathrm{p}$. 
Après cette épreuve, il est contraint de s'arrêter de travailler. II obtient une faible pension d'invalidité, qu'il touche chaque trimestre. Et malgré ses demandes de reconversion professionnelle auprès de la COTOREP $^{14}$, il se voit refuser le droit de travailler à nouveau. Encore une fois, son cas n'est pas singulier. Beaucoup de travailleurs immigrés n'ont pas eu droit à des formations pendant leur vie professionnelle, y compris après avoir été victime d'un accident du travail.

L'impasse médico-socio-professionnelle où se trouve Amin va provoquer de graves difficultés sociales et familiales. Elle entraîne ce que Yves, médecin du travail, appelle II des syndromes de rupture, d'abord dans le corps, puis du projet migratoire d'insertion sociale. II est rompu par l'accident du travail. Tous les sacrifices consentis par le migrant n'ont plus de raison d'être. Comme professionnel de la santé, on repère alors des conséquences psychologiques et familiales graves 11.

\section{La crise familiale et sa résolution}

Ce changement de situation a beaucoup de répercussions sur l'équilibre de la famille : non seulement il affecte son budget, mais il rend les rapports entre le mari, la femme et les enfants très tendus. Amin se sent inutile. II est très sombre, devient dépressif, parfois violent. II se sent dévalorisé en tant qu'homme. II repense avec nostalgie à son pays d'origine. Situation inextricable. Mais en recevant l'autorisation médicale de se rendre à nouveau au Maroc, Amin retrouve un peu de joie de vivre. II est parti pour restaurer une ruine, pour donner un toit à sa famille lorsqu'ils viendraient en vacances. Arrivé dans son village, Amin revoit ses amis et leur fait part de ses états d'âme. Pour lui changer les idées et comme remède, l'un d'eux, intéressé, lui propose la bigamie alors que lui-même n'est pas polygame. II lui présente Habiba qu'Amin épouse sur le champ puisque avoir une deuxième femme est considéré par certains musulmans comme un attribut de virilité. De son côté, l'ami entremetteur a ainsi l'espoir de pouvoir profiter des allers-retours d'Amin entre la France et le Maroc, où réside sa deuxième épouse, afin d'obtenir des biens de consommation introuvables dans son village.

Amin n'est pas le seul ouvrier marocain dans ce cas. Dans une recherche que Lamia Missaoui a menée en France et au Maghreb, elle a découvert le même comportement de la part d'hommes se trouvant

14. Commission technique d'orientation et de reclassement professionnel. 
dans l'incapacité d'exercer officiellement un emploi en France ${ }^{15}$. Comme Amin, ceux-ci étaient fiers d'être bien considérés professionnellement. Après avoir été victime d'un accident du travail, la société leur a renvoyé l'image d'assistés. Pour échapper à cette stigmatisation, ils ont retissé des liens avec leur région d'origine. Certains, comme Amin, sont devenus bigames. Petit à petit, ils se sont reconstruits socialement dans leur village et sont devenus des notables.

En France, c'est la crise, Diamila et ses enfants n'acceptent pas qu'Amin ait pris une seconde épouse. Diamila est mise devant un dilemme : divorcer ou accepter. Elle en parle à l'assistante sociale qui s'occupe de la famille, qui lui conseille de se séparer d'Amin. Elle prend la décision inverse: II'ai voulu, explique-t-elle, que mes enfants continuent à vivre avec leur père, qu'il ne les laisse pas II. Elle négocie un nouveau type de rapport avec son mari : elle ne divorcera pas mais exige de prendre en main l'organisation du ménage puisqu'il retourne au Maroc tous les deux mois. Elle qui sortait peu, se charge dorénavant avec l'aide de sa fille aînée des tâches administratives: "Chaque fois qu'il est nécessaire pour l'un de mes enfants que i'aille parler avec son professeur, ou qu'il doit aller chez le médecin, ou qu'il doit se présenter pour trouver un travail, je l'accompagne. Je me renseigne, je ne laisse jamais tomber. Je suis derrière lui II.

Parallèlement, Djamila s'investit au sein de groupes de parents et de professionnels qui réfléchissent ensemble aux moyens de prévenir les risques d'échec scolaire, de toxicomanie, de chômage encourus par les jeunes du quartier ${ }^{16}$. L'épreuve de la bigamie, contrairement aux apparences, a renforcé chez Djamila sa capacité à être actrice de sa propre vie, à devenir influente dans la sphère privée et publique. En arrivant en France, pendant dix ans elle a connu une période de bonheur conjugal auquel son mariage forcé ne l'avait pas a priori destinée. Elle a su négocier avec Amin le statut de partenaire, obtenu pour leurs enfants la liberté de choisir leur futur conjoint. Malgré les obstacles, elle a su retrouver par le dialogue avec son mari un certain équilibre familial important pour la réussite de leurs enfants. La crise familiale a donc consolidé leur couple parental, mais en mettant entre parenthèses leur couple conjugal.

15. Cf. MISSAOUI, Lamia, "Généralisation du commerce transfrontalier : petit ici, notable là-bas", Revue Européenne des Migrations Internationales, vol. 11, n 1, 1995, pp. 53-73.

16. Cf. DELCROIX, Catherine, "Transmission de l'histoire familiale et de la mémoire historique face à la précarité", Migrations Société, vol. 21, n 123-124, mai-août 2009, pp. 143-157. 
Suivre dans la durée un couple singulier confronté aux mêmes types de problèmes que de nombreux autres ménages permet de décrire l'action en mouvement, notamment de découvrir le travail constant de redéfinition des rapports de genre entrepris ici par Diamila avec Amin. Cet exemple particulier montre un processus plus général : les rapports entre les membres des familles immigrées changent constamment, comme d'ailleurs dans toutes les familles, autochtones ou pas. II faut en finir avec un certain fixisme, qui accompagne généralement le regard qu'une société porte sur l'Autre. En effet, l'Autre est aussi susceptible de changement que soi-même. Adopter un point de vue dynamique permet d'échapper à II une analyse culturelle du genre [qui] produit parfois des images statiques qui ne sont pas moins déterministes que les explications biologiques des rôles masculins/féminins dans la société $11{ }^{17}$.

Maintenir la famille en équilibre entre "ici" et "là-bas" pour assurer le devenir des enfants est un objet de débats constants et souvent de conflits entre époux. C'est de leur capacité à résoudre les crises familiales engendrées par l'exploitation et la précarité de leurs conditions d'existence que dépendent aussi en partie les rapports que les enfants entretiennent avec la France. En effet, les résultats des enquêtes menées montrent une différence d'appréciation entre les parents et les enfants à propos de leur expérience. Les adultes, arrivés en France en tant qu'immigrés marocains, se perçoivent comme privilégiés par rapport aux membres de leur famille restés au Maroc, en particulier en ce qui concerne la gratuité de l'école et la qualité des soins de santé. C'est ce sentiment qui explique leur patience face à la précarité. II leur donne la force et le calme nécessaires pour rechercher les solutions qui sont à leur portée. Les enfants, qui se comparent aux jeunes de leur génération, n'ont pas du tout ce point de vue. Ils se sentent discriminés et craignent que leur avenir ne soit bouché parce que descendants de parents immigrés et issus de milieux défavorisés.

$\grave{A}$ travers ces quelques exemples des dynamiques conjugale et familiale des couples marocains venus fonder une famille en France dans les années 1960-1970 apparaît l'obligation pour eux de ne pas opposer des traditions figées à une "modernité" omnipotente ; ils tentent plutôt, en se basant sur des évaluations de leurs expériences et de celles de leurs proches, de prendre là où ils les trouvent les éléments qui leur permettent d'avancer.

17. NADER, Laura, "Orientalisme, occidentalisme et contrôle des femmes", Nouvelles Questions Féministes, vol. 25, $\mathrm{n}^{\circ}$ 1, 2006, pp. 12-23 (voir p. 23). 